\section{Orientaciones éticas para la toma de decisiones médicas en el contexto de la pandemia de COVID-19 en Chile}

\author{
LUCA VALERA ${ }^{1,2}$, MARÍA ALEJANDRA CARRASCO ${ }^{1,2}$, \\ RODRIGO LÓPEZ ${ }^{1,3}$, PAULINA RAMOS ${ }^{1}$, \\ ROMMY VON BERNHARDI ${ }^{1,4}$, PAULA BEDREGAL ${ }^{1,5}$, \\ ALEJANDRA FLORENZANO ${ }^{1,6}$, IVÁN PÉREZ ${ }^{1,6}$, PATRICIA OLIVARES ${ }^{1,6}$, \\ IVONNE VARGAS ${ }^{7}$, XIMENA GONZÁLEZ ${ }^{1,7}$, PAULO LÓPEZ $^{1,8}$, \\ GLORIA DURÁN ${ }^{1}$, CONSTANZA RICHARDS ${ }^{1}$, RICARDO CASTRO ${ }^{1,9}$
}

\section{Ethical guidelines for medical decision-making during COVID-19 pandemic in Chile}

The catastrophic emergency experienced by many countries with the COVID-19 pandemic emphasized the importance of bioethics for decision-making, both at the public health (equitable and effective policies) and at the clinical level. At the clinical level, the issues are the fulfillment of medical care demand with adequate health care teams, infrastructure, and supplies, and to cover critical care demands that surpass the available resources. Therefore, ethically correct approaches are required for the allocation of life sustaining resources. There are recommendations for the allocating life support during disasters based on multiple considerations, including ethical ones. However, the ethical criteria of existing guidelines are variable. Ethical principles usually considered are saving the greatest number of lives, saving the greatest number of years of life and the principle of the life cycle or the goal to give each individual equal opportunity to live through the various phases of life. However, the centrality of the human being and the search for the common good should be considered. Knowledge of public perspectives and moral benchmarks on these issues is essential. A successful assignment effort will require everyone's trust and cooperation. Decision making should be planned and discussed in advance, since in-depth deliberation will be extremely complex during the disaster. Our goal is to help the health care teams to wisely allocate resources in shortage periods.

(Rev Med Chile 2020; 148: 393-398)

Key words: Ethics, Clinical; Health Planning Guidelines; Resource Allocation.
${ }^{1}$ Centro de Bioética, Facultad de Medicina, Pontificia Universidad Católica de Chile. Santiago, Chile. 2Instituto de Filosofía, Pontificia Universidad Católica de Chile. Santiago, Chile.

${ }^{3}$ Departamento de Anestesia,

Facultad de Medicina, Pontificia Universidad Católica de Chile.

Santiago, Chile.

${ }^{4}$ Departamento de Neurología, Facultad de Medicina, Pontificia Universidad Católica de Chile.

Santiago, Chile.

${ }^{5}$ Departamento de Salud Pública, Facultad de Medicina, Pontificia Universidad Católica de Chile.

Santiago, Chile.

${ }^{6}$ Departamento de Medicina Interna, Facultad de Medicina,

Pontificia Universidad Católica de Chile. Santiago, Chile.

${ }^{7}$ Escuela de Enfermería, Pontificia Universidad Católica de Chile.

Santiago, Chile.

${ }^{8}$ Facultad de Teología, Pontificia Universidad Católica de Chile.

Santiago, Chile.

9Departamento de Medicina Intensiva, Pontificia Universidad Católica de Chile. Santiago, Chile.

Recibido el 26 de marzo de 2020 aceptado el 28 abril de 2020 .

Correspondencia a:

Luca Valera

Diagonal Paraguay 362, Edificio Académico de Medicina luvalera@uc.cl

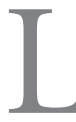

a rápida propagación de la enfermedad por coronavirus 2019, Covid-19 (acrónimo del inglés coronavirus disease 2019), producido por el coronavirus 2 del síndrome respiratorio agudo grave (SARS-CoV-2), a nivel mundial, está creando escenarios complejos para la toma de decisiones médicas, las que están influenciadas, muy frecuentemente, por la escasez de recursos clínicos y de profesionales de la salud ${ }^{1}$. En este sentido, el "caso italiano" es particularmente dramático, y nos obliga a reflexionar en cómo se deben realizar los juicios éticos en un contexto 
excepcional ${ }^{1,3}$. Las preguntas éticas que surgen son muchas, incluyendo el sentido y el impacto de las restricciones de desplazamiento o de actividades, el cierre de colegios, el cierre de fuentes laborales, el aislamiento social, la intromisión en la privacidad de las personas contagiadas y sus contactos, e incluso la investigación en humanos: todas decisiones relevantes para la salud pública, pero que impactan de manera muy desigual a los grupos más vulnerables. En el ámbito clínico, las dudas éticas más punzantes se refieren principalmente a la asignación de los recursos en un contexto excepcional de "medicina de catástrofes" y, en consecuencia, a la admisión a la UCI y al manejo de los pacientes que cursan una enfermedad particularmente grave ${ }^{4-6}$. Esta situación convoca a los equipos de la salud y a los expertos en ética clínica a plantear cuales son los criterios éticos que se usan -o que se deben usar- en estas circunstancias ${ }^{7-9}$.

Como Centro de Bioética, y con el apoyo de académicos de la Facultad de Medicina de la Pontificia Universidad Católica de Chile, planteamos algunos principios y valores que consideramos importante resguardar en la toma de decisiones del equipo médico en el contexto antes descrito. Este documento busca aportar una mirada ética $-\mathrm{y}$ no exclusivamente técnica-para esbozar un marco general que apoye a los equipos de salud en la toma de decisiones en estas condiciones excepcionales de gran desequilibrio entre las necesidades y los recursos disponibles.

Para ello, se enumeran algunas orientaciones básicas para una situación de crisis del sistema sanitario como la pandemia que estamos viviendo. No se pretende dar respuestas concretas a todas las dudas éticas particulares que puedan surgir, pero sí lineamientos capaces de orientar y apoyar el juicio prudencial del equipo médico. Es importante comprender que estos son contextos de decisión altamente variables (por la progresión de la difusión del Covid-19 en el país, por sus posibles mutaciones y el mayor conocimiento del comportamiento del virus que se pueda ir adquiriendo, así como la escasez de evidencia científica ${ }^{10}$ ), por lo que será necesario contextualizar y adecuar las orientaciones a la situación clínica de cada paciente y de cada hospital o clínica.

Hay que considerar que distintas organizaciones, centros de estudio y gremios, entre otros, tanto a nivel mundial como regional y nacional, han formulado recomendaciones $\mathrm{u}$ orientaciones éticas para abordar una posible crisis sanitaria $^{11-20}$. Algunas de ellas nos han inspirado para la formulación de nuestras recomendaciones. Sin embargo, lo esencial y original de este documento está, a nuestro modo de ver, en que el principio fundamental que se busca salvaguardar en cada una de estas recomendaciones y en el conjunto de ellas, es la centralidad de la persona en la relación y la atención clínica, junto con la consideración por el bien común. Este principio, fundamento del ethos de la medicina, obliga consistentemente a:

- Tratar a cada paciente acorde a su dignidad, en cuanto persona humana;

- Ofrecer un cuidado de calidad, proporcionado a la condición de cada paciente, reconociendo las limitaciones que impone la escasez de recursos;

- Cuidar al equipo de salud particularmente expuesto en esta situación, desarrollando una conciencia de responsabilidades compartidas.

\section{Presentación de las orientaciones éticas}

Las orientaciones están agrupadas en cuatro secciones, según el tipo de decisión al que se refieren (admisión a la UCI, tratamiento en la UCI, sobre el equipo clínico y sobre la gestión del centro sanitario). Están, también, redactadas de modo breve e imperativo, para mayor claridad. Si bien cada una de ellas tiene una sólida fundamentación ética, en este trabajo no se puede explicitar por motivos de disponibilidad de espacio y que, por esta misma razón, no se referirán a trabajos publicados precedentemente.

\section{Orientaciones éticas}

\section{I) Sobre los criterios para admisión de paciente a la UCI}

1. Más que nunca en el contexto de pandemia, vale el principio del to care por sobre to cure (cuidar por sobre curar).

- A pesar de la escasez de recursos, no se puede renunciar a cuidar a cada paciente, es decir, no se puede abandonar a ninguna persona. Todo paciente tiene derecho a ser informado de cómo se lo está cuidando ("no-abandonando"). 
2. La admisión de todos los pacientes debe ser considerada de manera equitativa. Los criterios se aplican a todos ellos, sin discriminar ni positiva ni negativamente a los infectados por Covid-19.

3. En situaciones de crisis, los criterios de admisión y alta deben ser flexibles y se deben adaptar localmente según la disponibilidad de recursos y de personal, la posibilidad real de transferir pacientes, el número de ingresos en curso o planeado.

4. La presencia de comorbilidades y estado funcional previo debe evaluarse cuidadosamente. En esta valoración, no se deben tomar decisiones médicas exclusivamente con criterios técnicos o económicos, sino hacer lo que es proporcionado a la condición clínica del paciente en un contexto de escasez de recursos.

- Las expectativas de recuperación deben balancearse con la condición que tenía el paciente, previa a caer en una condición crítica. Por lo mismo, es necesaria una valoración contextual de las posibilidades de recuperación, así como de la posible futilidad de tratamientos y monitoreo habitualmente costosos.

- La edad como criterio para ingresar a la UCI solo debe ser una referencia para definir el estado de salud y pronóstico del paciente. Los límites de edad no deben ser un criterio en sí mismo, ni mucho menos el único criterio dirimente.

5. Cuando corresponda, la posibilidad del ingreso a la UCI debe discutirse con el paciente y su familia lo antes posible.

- Esto debiese comenzar a definirse desde el servicio de urgencias. Debe recogerse la opinión expresada por el paciente, de existir, e informarle respecto de lo eventualmente inadecuado de ingresar a la UCI.

- Si es posible, se debe identificar con antelación cuáles son los pacientes para los que se considera proporcionado el ingreso a Cuidados Intensivos si ocurriera deterioro clínico. Esta decisión debe ser informada a los distintos equipos a cargo del paciente.

6. Toda indicación de "no intubar" o "no reanimar" debe estar registrada en la ficha clínica.

- De este modo puede ser utilizada como guía si el deterioro clínico ocurre precipita- damente y en presencia de cuidadores que no han participado en la planificación de la atención o no conocen al paciente.

- En este sentido, es importante velar por una comunicación fluida y transparente entre los distintos cuidadores (incluso los familiares).

7. Respecto de los pacientes para quienes se considere desproporcionado el manejo en UCI, la decisión de adecuar el esfuerzo terapéutico debe estar fundamentada, comunicada y documentada.

- Dicha adecuación no excluye el uso de terapia antimicrobiana, por ejemplo, u otros tratamientos de una complejidad más baja.

- Se debe asegurar en todo momento el control de síntomas de malestar físico como son el dolor o la disnea, así como el acompañamiento.

- Se debe considerar que, dependiendo tanto del progreso de la pandemia como de la escasez de recursos, la proporcionalidad o desproporcionalidad de los tratamientos puede variar, requiriéndose una evaluación dinámica. Es decir, las medidas que se consideren ordinarias en un cierto momento y lugar (por existencia de recursos y de personal), en otro contexto pueden considerarse extraordinarias (por escasez de estos), y por tanto no éticamente obligatorias.

- Conviene que esta decisión se tome en conjunto con quien tenga una visión más general de la situación de la pandemia y, de ser posible, con el comité de ética asistencial o expertos en ética.

\section{II) Sobre los tratamientos de pacientes en UCI}

8. Todos los ingresos a Cuidados Intensivos deben estar sujetos a reevaluación periódica y constante de los objetivos y la proporcionalidad de los tratamientos.

- Si se considera que un paciente tiene mal pronóstico por no mostrar una respuesta favorable al tratamiento en curso, habiéndose agotado las propuestas curativas, no debe posponerse la decisión de disminuir la terapia y una reasignación de Cuidados Intensivos a paliativos, en un escenario de afluencia excepcionalmente alta de pacientes. 
9. Se debe procurar una muerte digna para los pacientes que fallezcan por infección Covid-19, así como por cualquier otra causa.

- El proceso de fin de vida en una UCI conlleva hacerse cargo de aspectos físicos, psicológicos, espirituales, sociales y familiares.

- Se entiende por muerte digna aquella muerte que ocurre debido al avance natural de la enfermedad y en la que se intenta proporcionar el máximo de confort, alivio del dolor y de otros síntomas asociados a la agonía. No puede considerarse digna una muerte en la que terceros decidan adelantar el proceso natural aduciendo razones económicas, de efectividad o incluso supuestamente humanitarias para aliviar el dolor o el sufrimiento.

- Si se definió previamente no ingresar a un paciente a la UCI o, estando en esta unidad, se ha definido no avanzar en la intubación y conexión a ventilación mecánica, en ningún caso significará el abandono del paciente, persistiendo el deber de otorgarle cuidados básicos y tratamientos proporcionados, incluyendo los cuidados paliativos. Si se prevé un período agónico largo, se debe evaluar trasladar el paciente fuera de la UCI, ya que esto puede ayudar a disminuir el estrés de los profesionales de UCI y de los familiares del paciente.

- Hay que considerar que, por motivos de seguridad para el equipo médico, para los familiares o cercanos del paciente y para la población general, puede ser necesario prohibir las visitas y el acompañamiento en los momentos finales de la vida del paciente. Velar por la muerte digna significará también buscar medidas alternativas para que el paciente y sus seres queridos puedan gozar de alguna forma de acompañamiento psicológico y espiritual.

10. Si surgen situaciones de dificultad particular durante el proceso de toma de decisiones, se debe buscar la segunda opinión de otros médicos con más experiencia, del comité de ética asistencial o de algún experto en ética clínica.

- Hay que intentar construir redes entre los comités de ética asistencial (extendiendo a los expertos en ética clínica), entre clínicos y eticistas, para generar un apoyo constante y eficiente y para que los equipos clínicos no se sientan abandonados en decisiones particularmente difíciles.

11. La decisión de adecuar los cuidados intensivos debe ser discutida y compartida tanto como sea posible por el equipo tratante $y$, en la medida de lo posible, con el paciente y su familia.

- Se recomienda, también, que todas las familias de pacientes ingresados a UCI donde existan casos de Covid-19 reciban la información habitual diaria que proporciona el equipo médico fuera de la unidad, previa designación de un interlocutor responsable. Este comunicará al resto de los cercanos las novedades respecto del manejo médico y la evolución del paciente, con el fin de descomprimir al equipo médico de la necesidad de entregar varias las veces información de un mismo paciente a diferentes familiares o cercanos. En caso de que la situación en el hospital se vuelva muy crítica, puede recurrirse a canalizar la información por vía exclusivamente telefónica.

12. La decisión de adecuar los Cuidados Intensivos debe estar fundada en razones, debe poder comunicarse.

- Un criterio para averiguar si es posible tomar una decisión tan importante como esta es si hay razones claras para hacerlo. Una evidencia de la existencia de estas razones es que se pueden comunicar.

- Es esperable que la necesidad de tomar decisiones repetidas de este tipo hará que el proceso de toma de decisiones sea más sólido y adaptable a la disponibilidad de recursos en cada UCI.

\section{III) Sobre el equipo clínico}

13. El centro clínico deberá siempre proporcionar los elementos de protección personal según las mejores recomendaciones estándar para estos casos, de modo que el trabajo del equipo tratante no implique un riesgo innecesario para su salud ni la de sus familiares.

- Cada funcionario (profesionales médicos, enfermeros, kinesiólogos, técnicos de enfermería, entre otros) tiene el deber de valorar y presentar oportunamente sus vulnerabilidades personales ante su jefatura para tomar las medidas pertinentes. 
14. Cuando las condiciones lo permitan, se debe dedicar tiempo y recursos a la evaluación y monitoreo del burnout, el estrés y la angustia moral de los profesionales sanitarios.

- La experiencia en el hemisferio norte respecto del impacto de la infección por Covid-19 constituye un elemento valioso para anticipar las principales áreas en las cuales el personal sanitario puede verse afectado por esta pandemia.

- Conviene establecer y fortalecer redes para el intercambio de información entre centros y profesionales en cuanto a las experiencias del personal de salud, y diseñar así estrategias que permitan prevenir y mitigar el impacto de estas situaciones.

- Hay que velar por que cada miembro del equipo se sienta siempre apoyado por los demás, y que todos se sientan responsables de las decisiones compartidas que se hayan tomado, cada uno según el papel que desempeña.

- Hay que favorecer la realización de ritos convenidos por el equipo (pausa de silencio, debriefing, etc.) y de disponer espacios de contención y conversación posteriores al fallecimiento de un paciente. Estas prácticas deben ser alentadas y mantenidas, aunque la contingencia eventualmente las restrinja a un formato más abreviado y menos concurrido.

- Es necesaria la reflexión en torno al ethos profesional, al cuidado de los pacientes y al autocuidado. Crisis como ésta no deben impedir recordar cuál es la verdadera vocación de todas las personas que trabajan en el cuidado del paciente.

\section{IV) Sobre la gestión del hospital/clínica}

15. Para quienes dirigen los hospitales/clínicas, es un deber planificar lo antes posible la coordinación de todos los miembros y de los equipos de salud a corto y largo plazo.

- Dicha planificación tiene que hacerse con todos los equipos e informar con tiempo a cada miembro el papel que cumplirá (turnos, cambios de funciones, reemplazos, etc.).

- Es aconsejable que se genere un claro liderazgo para articular todos los sub-equipos que se constituirán ad hoc y conducir las acciones de preparación y coordinación de la crisis. Cada institución deberá buscar el modelo más apropiado según su realidad local.
16. Las decisiones que se tomen a nivel directivo del centro sanitario sobre la gestión de los recursos y los criterios éticos que se utilizarán durante la crisis deben ser comunicadas a todo el equipo de salud, de modo de crear una "ética corporativa".

- Todos los funcionarios del centro sanitario, particularmente los equipos de salud, deben estar informados (y participar) del proceso de toma de decisiones en tiempos de crisis. Se requiere transparencia, comunicación, y explicar el sentido de las medidas.

- Debe existir un acuerdo previo sobre cómo comunicar las decisiones (terminología que se usará, quiénes tendrán esa tarea, tiempos, etc.), tanto dentro del centro de salud, como a los pacientes y sus familiares.

- Las instituciones deben considerar el cuidado de los profesionales de la salud una prioridad, otorgándoles los medios materiales y sustento psicológico y humano necesario, pues son ellos quienes cuidan a la población.

Agradecimientos: Se agradece al Prof. Ricardo Moreno Mauro, el Dr. Arturo Grau Diez y la Dra. Paula Cox Cruzat por sus sugerencias.

\section{Referencias}

1. Lowe A, Hewlett A, Schonfeld T. How Should Clinicians Respond to International Public Health Emergencies? AMA J Ethic 2020; 22 (1): E16-21.

2. Rosenbaum L. Facing Covid-19 in Italy - Ethics, Logistics, and Therapeutics on the Epidemic's Front Line. NEJM 2020; doi: 10.1056/NEJMp2005492.

3. Mounk Y. The extraordinary decisions facing Italian doctors. Atlantic. 2020. Disponible en: https://www. theatlantic.com/ideas/archive/2020/03/who-gets-hospital-bed/607807/ [Consultado el 15 de marzo de 2020].

4. Daugherty Biddison EL, Gwon HS, Schoch-Spana M, Regenberg AC, Juliano C, Faden RR, Toner ES. Scarce Resource Allocation During Disasters: A Mixed-Method Community Engagement Study. Chest 2018; 153 (1): 187-95.

5. Daugherty Biddison EL, Faden R, Gwon HS, Mareiniss DP, Regenberg AC, Schoch-Spana M et al. Too many patients... a framework to guide statewide allocation of scarce mechanical ventilation during disasters. Chest 2019; 155: 848-54. 
6. Emanuel EJ, Persad G, Upshur R, Thome B, Parker M, Glickman A et al. Fair Allocation of Scarce Medical Resources in the Time of Covid-19. NEJM. Doi: 10.1056/ NEJMsb2005114.

7. Sokol D. The life and death decisions of covid-19. The BMJ opinion. 2020. Disponible en: https://blogs.bmj. com/bmj/2020/03/20/daniel-sokol-the-life-and-deathdecisions-of-covid-19/ [Consultado el 22 de marzo de 2020].

8. Ives J. Coronavirus may force UK doctors to decide who they'll save. The Guardian. 2020. Disponible en: https:// www.theguardian.com/commentisfree/2020/mar/14/ coronavirus-outbreak-older-people-doctors-treatment-ethics. [Consultado el 23 de marzo de 2020].

9. Gandhi R, Patel A. What if Two COVID-19 Victims Need Ventilators and Just One Is Available? Scientific American. 2020. Disponible en: https://blogs.scientificamerican.com/observations/what-if-two-covid-19victims-need-ventilators-and-just-one-is-available/ [Consultado el 23 de marzo de 2020].

10. Salajan A, Tsolova S, Ciotti M, Suk JE. To what extent does evidence support decision making during infectious disease outbreaks? A scoping literature review. Evidence \& Policy 2020; 16(1). Doi: 10.1332/17442642 0X15808913064302.

11. The Nuffield Council on Bioethics. 2020. New briefing: Responding to the COVID-19 pandemic - ethical considerations. 2020. Disponible en: https://www.nuffieldbioethics.org/assets/pdfs/Ethical-considerations-in-responding-to-the-COVID-19-pandemic.pdf [Consultado el 23 de marzo de 2020].

12. Sociedad Española de Anestesiología, Reanimación y Terapéutica del dolor (SEDAR). Marco ético pandemia COVID-19. 2020. Disponible en: https://www.sedar.es/ images/site/NOTICIAS/coronavirus/Marco_etico_coronavirus.pdf [Consultado el 23 de marzo de 2020].

13. Società Italiana di Anestesia Analgesia Rianimazione e Terapia Intensiva (SIAARTI). Clinical ethics recommendations for the allocation of intensive care treatments, in exceptional, resource-limited circumstances. 2020. Disponible en: http://www.siaarti.it/SiteAssets/News/COVID19\%20-\%20documenti\%20SIAARTI/SIAARTI\%20 -\%20Covid-19\%20-\%20Clinical\%20Ethics\%20Reccomendations.pdf [Consultado el 17 de marzo de 2020].

14. Hick JL, Hanfling D, Wynia MK, Pavia AT. National
Academy of Medicine. 2020. Duty to Plan: Health Care, Crisis Standards of Care, and Novel Coronavirus SARS-CoV. Disponible en: https://nam.edu/duty-toplan-health-care-crisis-standards-of-care-and-novelcoronavirus-sars-cov-2/ [Consultado el 24 de marzo de 2020].

15. Comisions Deontológiques del Consell de Collegis de Metges de Catalunya (CCMC). Consideraciones deontológicas en relación a la pandemia Covidien-19. 2020. Disponible en: https://www.comb.cat/Upload/ Documents/8747.PDF [Consultado el 23 de marzo de 2020].

16. Comité Consultatif National d'Éthique. Contribution du comité consultatif national d'éthique: Enjeux éthiques face à une pandémie. (Contribución de la comisión consultora nacional de ética: Problema éticos ante una pandemia). 2020. Disponible en: https://www.ccne-ethique.fr/sites/default/files/reponse_ccne_-_covid-19_def. pdf [Consultado el 24 de marzo de 2020].

17. Swiss Academy of Medical Sciences. COVID-19 pandemic: triage for intensive-care treatment under resource scarcity. 2020. Disponible en: https:/www.samw.ch/ en/Ethics/Topics-A-to-Z/Intensive-care-medicine.html [Consultado el 24 de marzo de 2020].

18. Berlinger N, Wynia M, Powell T, Hester DM, Milliken A, Fabi R et al. The Hastings Center. Ethical Framework for Health Care Institutions Responding to Novel Coronavirus SARS-CoV-2 (COVID-19). Guidelines for Institutional Ethics Services Responding to COVID-19 Managing Uncertainty, Safeguarding Communities, Guiding Practice. 2020. https://www.thehastingscenter. org/ethicalframeworkcovid19/ [Consultado el 24 de marzo de 2020].

19. Sociedad Chilena de Medicina Intensiva (SOCHIMI). Recomendaciones generales respecto de las decisiones éticas difíciles y la adecuación de la intensidad asistencial e ingreso a unidades de paciente crítico en situaciones de crisis. Disponible en: https://www.medicina-intensiva. cl/site/covid/recomenda_etica.pdf [Consultado el 23 de marzo de 2020].

20. Wax RS, Christian MD. Practical recommendations for critical care and anesthesiology teams caring for novel coronavirus (2019-nCoV) patients. Can J Anesth/J Can Anesth (2020). https://doi.org/10.1007/s12630-02001591-x. 